\title{
REPORT ON CONTINUOUS CURVES FROM THE VIEWPOINT OF ANALYSIS SITUS*
}

\author{
BY R. L. MOORE
}

1. The Characterization of a Continuous Curve. A continuous curve is the set of points represented by the pair of equations

$$
x=f_{1}(t), \quad y=f_{2}(t),
$$

where $f_{1}(t)$ and $f_{2}(t)$ are continuous functions of $t$ on the interval $I:(0 \leqq t \leqq 1)$. Thus a continuous curve is the image of the straight line interval $I$ under a continuous $\dagger$ transformation which transforms each point $X$ of the interval $I$ into a single point $T(X)$ of the curve. In case this transformation does not throw any two distinct points of $I$ into the same point of the curve $T(I)$, then $T(I)$ is a simple continuous arc. Thus a simple continuous arc is in one to one continuous correspondence $\ddagger$ with a straight line interval. If $T(0)=T(1)$, but no point of $T(I)$ except $T(0)$ is the transform, under $T$, of more than one point of $I$, then $T(I)$ is a simple closed curve. It easily follows that a simple closed curve is in one to one continuous correspondence with a circle.

It is clear from the above definition that every continuous

* Presented before the Southwestern Section of the Society, in somewhat different form, at the Symposium held in Lawrence, Kansas, Dec. 2, 1922.

$\dagger$ The point $P$ is said to be a limit point of the point set $M$ if, for every positive number $\epsilon$, there are points of $M$, distinct from $P$, at a distance from $P$ less than $\epsilon$. A transformation $T$ which throws a point set $M$ into a point set $T(M)$ is said to be continuous if, in case the point $P$ of $M$ is a limit point of a point set $N$ which is a subset of $M$, then $T(P)$ is a limit point of $T(N)$.

$\ddagger$ Two point sets $M$ and $N$ are said to be in one to one correspondence if there exists a correspondence in which (a) to each point $P$ of $M$ there corresponds just one point $P^{\prime}$ of $N,(b)$ no two distinct points of $M$ correspond to the same point of $N,(c)$ if the point $P^{\prime}$ of $N$ corresponds to the point $P$ of $M$, then the point $P$ of $M$ corresponds to the point $P^{\prime}$ of $N$ and conversely. Such a correspondence is sometimes called a one to one reciprocal correspondence. For so-called one to one correspondences which are not reciprocal I prefer to use the term transformation which seems to me to be more suggestive of an operation which is thought of as taking place in one direction. 
curve is a bounded continuum.* That it is not, however, very easy to determine directly from this definition whether or not even some of the simplest continua are continuous curves will, I think, be admitted if one recalls the interest aroused by Peano's discovery of the fact that a square plus its interior is such a curve and the proofs of this fact that were given by Peano, Hilbert and E. H. Moore. $\dagger$

In 1908, Schoenflies established a theorem which embodies a point set theoretic characterization (definition) of a continuous curve. This is, apart from minor matters of phraseology, etc., as follows: In order that a bounded continuum $M$ should

* A set of points is said to be closed if it contains all its limit points. A point set is said to be connected if, however it be divided into two mutually exclusive subsets, one of them contains a limit point of the other one. (Cf. N. J. Lennes, American Journal, vol. 33 (1911), pp. 287-326.) A set of points is said to be bounded if it lies wholly in the interior of some circle. A continuum is a closed and connected point set.

$\dagger$ Peano, Sur une courbe, qui remplit toute une aire plane, Mathematische Annalden, vol. 36 (1890); Hilbert, Ueber die stetige Abbildung einer Linie auf ein Flächenstück, ibid., vol. 38 (1891), pp. 459-460; E. H. Moore, On certain crinkly curves, Transactions of this Society, vol. 1 (1900), pp. 72-90. That a square plus its interior is not a simple continuous arc had been proved by Netto about ten years before. (E. Netto, Beitrag zur Mannigfaltigkeitslehre, Journal FÜR MAthematik, vol. 86 (1879), pp. 263-268.) On page 329 of the 1907 edition of his The Theory of Functions of a Real Variable, Hobson gives a proof of this theorem. Referring to the proofs of Netto and of Loria, he says: "In the proof given by these writers it is assumed that a closed curve corresponds to a linear sub-interval of $(0,1)$; this is not necessarily the case, for a non-dense closed set may correspond to the closed curve." This statement of Hobson's (which is repeated in the 1921 edition of his treatise) is manifestly incorrect. Neither a simple closed curve nor any other sort of connected point set could possibly be in one to one continuous correspondence with a non-dense closed set or any other set which is not connected. As far as I know, I have never seen anywhere in the literature the following extremely simple proof of Netto's theorem. Let $X$ denote a point, other than an end-point, of the interval $I$. Suppose there exists a continuous one to one correspondence between $I$ and the point set $K$ constituted by a square plus its interior. Let $X^{\prime}$ denote the point of $K$ which corresponds to $X$. The set $I-X$ is not connected. Hence $K-X^{\prime}$ is not connected. But clearly $K-X^{\prime}$ is connected. Thus the supposition that the correspondence in question exists leads to a contradiction. The same proof shows that there is no one to one continuous correspondence between the interval $I$ and the set of points constituted by a simple closed curve. 
be a continuous curve it is necessary and sufficient that (a) for every given positive number $\epsilon$ there are not more than a finite number of complementary domains * of $M$ of diameter greater than $\epsilon,(b)$ the boundary of every complementary domain of $M$ is accessible $\dagger$ at each of its points, from all sides, with respect to that domain.

That the same class of point sets would not be characterized by this definition if accessibility from all sides were replaced by mere accessibility may be seen with the help of the following example. Let $M_{1}$ denote the point set composed of the straight line intervals $A B, B C, C D$, where the coordinates of the points $A, B, C$, and $D$ are $(0,1),(0,-2),(1 / \pi,-2)$ and $(1 / \pi, 0)$ respectively. Let $M_{2}$ denote that portion of the curve $y=\sin (1 / x)$ which lies between the lines $x=0$ and $x=1 / \pi$. Let $M_{3}$ denote the point set $M_{1}+M_{2}$. The point set $M_{3}$ separates the plane into two domains $K$ and $H$, where $H$ is bounded. Let $M$ denote the continuum $H+M_{3}$. The only domain complementary to $M$ is $K$. Furthermore $M_{3}$, the

* A connected point set $K$ is said to be a domain if for each point $P$ of $K$ there exists a positive number $\delta_{P}$ such that $K$ contains every point whose distance from $P$ is less than $\delta_{P}$. The boundary of a point set $K$ is the point set composed of all points $[X]$ such that for each positive number $\epsilon$ there is at least one point of $K$ and at least one point which does not belong to $K$, at a distance from $X$ less than $\epsilon$. A complementary domain of a closed point set $M$ is a domain $K$ which contains no point of $M$ but whose boundary is a subset of $M$. The diameter of a bounded point set $K$ is a number $d$ such that $(a)$ if $X$ and $Y$ are any two points of $K$ then the distance from $X$ to $Y$ is not greater than $d$, (b) for every positive number $e$ there exist points $X$ and $Y$ belonging to $K$ such that the distance from $X$ to $Y$ differs from $d$ by less than $e$.

$\dagger$ The boundary $B$ of a domain $K$ is said to be accessible at the point $P$ with respect to $K$ (and $P$ is said to be accessible from $K$ ) if, for every point $A$ in $K$, there exists a simple continuous arc $A P$ which lies, except for the point $P$, wholly in the domain $K$. Suppose that $X Y Z$ is a simple continuous arc whose end-points $X$ and $Y$ lie on $B$, but every other point of which lies in the domain $K$. If $B$ is connected then $K$ is simply connected and is separated by the point set $X Y Z-(X+Y)$ into two mutually exclusive domains, $K_{1}$ and $K_{2}$, such that $K=X Y Z-(X+Y)+K_{1}$ $+K_{2}$. At least one of these domains (call it $D_{X Y Z}$ ) has the point $P$ on its boundary. If, for every arc $X Y Z$ satisfying the conditions stipulated, the point $P$ is accessible from $D_{X Y Z}, P$ is said to be accessible from $K$ from all sides and $B$ is said to be accessible at $P$, from all sides, with respect to $K$. 
boundary of $K$, is accessible at each of its points with respect to $K$. That it is not however accessible at every point from all sides with respect to $K$ may be seen as follows. Let $E$ denote the point $(1 / \pi, 2)$ and let $A E D$ denote the simple continuous arc composed of the straight line intervals $A E$ and $E D$. The origin $O$ is not accessible from both of the domains into which $K$ is divided by the point set $A E D-(A+D)$, although $O$ lies on the boundary of each of these domains. Thus $O$ is not accessible from all sides with respect to $K$. Hence, by Schoenflies' theorem, $M$ is not a continuous curve.

While Schoenflies' definition holds good for continuous curves in the plane, it does not hold for those in three dimensions. In fact I have recently shown * that, in order that, in space of three dimensions, a continuum $M$ should be a continuous curve, it is not sufficient that it should have the two properties, $(a)$ and $(b)$, stipulated in Schoenflies' definition and it is not necessary that it should have either of them.

In 1914 Hans Hahn showed that in order that, in space of any number of dimensions, a bounded continuum should be a continuous curve, it is necessary and sufficient that it should have a property which he designates as that of connectedness im kleinen. $\dagger$ A continuum is said to be connected im kleinen

* On the relation of a continuous curve to its complementary domains in space of three dimensions, Proceedings of the National Academy, vol. 8 (1922), pp. $33-38$. On page 38 of this paper there is a theorem, involving conditions concerning the complementary domains of a bounded continuum $M$, in space of three dimensions, which are sufficient, but not necessary, in order that $M$ should be a continuous curve.

$\dagger$ Hans Hahn, Über die allgemeinste ebene Punktmenge, die stetiges Bild einer Strecke ist, JAHREsBericht Der Vereinigung, vol. 23 (1914), pp. 318-322. See also, however, S. Mazurkiewicz, Sur les lignes de Jordan, Fundamenta Mathematica, vol. 1 (1920), pp. 166-209. In this paper Mazurkiewicz introduces the same conception and establishes the same result, and refers, in this connection, to earlier papers of his own as follows: $O$ arytmetyzacji kontinuow, C. R. Soc. Sc. VARsovie, vol. 6 (1913); $O$ arylmetyzacji kontinuow, II, ibid., vol. 6 (1913); O pewnej klasyfikacji punktow lezacych na kontinuach dowolnych, ibid., vol. 9 (1916). I do not have access to these papers. The conception of connectedness im kleinen, as applied to a simple closed curve, was used by Pia Nalli, in the paper Sopra una definizioni di dominio piano limitato da una curva continua, senza punti multipli, Rendiconti di Palermo, vol. 32 (1911), pp. 391-401. 
at the point $P$ if for every positive number $\epsilon$ there exists a positive number $\delta_{P_{\varepsilon}}$ such that if $X$ and $Y$ are any two points at a distance from $P$ less than $\delta_{P e}$ then they lie together in a closed and connected subset of $M$ every point of which is at a distance less than $\epsilon$ from the point $P$. This definition of a continuous curve has, for many purposes, a decided advantage over that of Jordan. For instance, it is easy to see that a square plus its interior (or, in three dimensions, a cube plus its interior) satisfies this definition and is therefore a continuous curve. Consider, on the other hand, the point sets $M_{1}, M_{2}$, $M_{3}$, described as follows:

Let $M_{1}$ denote the point set obtained by adding to the interval from $(0,-1)$ to $(0,1)$ that part of the curve $y=\sin$ $(1 / x)$ which lies to the right of the $y$-axis and is bounded on the right by the line $x=1$. Let $M_{2}$ denote the point set consisting of the intervals $O B, O B_{1}, O B_{2}, O B_{3}, \cdots$ where $O$ is the origin of coordinates, $B$ is the point $(0,1)$ and, for every $n, B_{n}$ is the point $(1 / n, 1)$. Let $M_{3}$ denote the point set obtained by adding to the set $M_{1}$ the set of all the intervals $A_{m n} B_{m n}$ where, for every pair of positive integers $m$ and $n$, $A_{m n}$ and $B_{m n}$ denote the points $(0, m / n)$ and $(m / n, m / n)$ respectively. I venture to surmise that one who is acquainted with no other characterization of a continuous curve than that of Jordan would probably not be able to decide, without some difficulty, the question, which of the sets $M_{1}$, $M_{2}, M_{3}$ is a continuous curve and which are not.* It is easy, however, to see that neither $M_{1}$ nor $M_{2}$ is connected im kleinen at the point $(0,1 / 2)$, while $M_{3}$ is connected im kleinen everywhere. The set $M_{3}$ is accordingly a continuous curve, while $M_{1}$ and $M_{2}$ are not.

Mazurkiewicz, Tietze, and I, working, as far as I know, entirely independently of each other, have shown $\dagger$ that, in

* For examples in space of three dimensions see my paper On the relation of a continuous curve to its complementary domains in space of three dimensions, loc. cit.

$\dagger$ S. Mazurkiewicz, loc. cit., H. Tietze, Ueber stetige Kurven, Jordansche Kurvenbogen und geschlossene Jordansche Kurven, Mathematische ZeitsCHRIFT, vol. 5 (1919), pp. 284-291; R. L. Moore, A theorem concerning 
order that a bounded continuum should be a continuous curve it is necessary that it should be arc-wise* connected. That this condition is not sufficient is shown by the existence of the above-described point set $M_{2}$, which, though arc-wise connected, is not a continuous curve. However, I have recently established the following theorem which embodies a generalization of the above-mentioned result.

THeorem A. In order that a continuum $M$ should be a continuous curve it is necessary that every maximal connected subset of an open subset of $M$ should be arc-wise connected. $\dagger$

Less than a week before the date of the delivery of this address, Mr. R. L. Wilder showed $\ddagger$ that this condition is also sufficient. It therefore affords a complete characterization of a continuous curve. It may be of interest to see just how this condition fails to hold true for the set $M_{2}$. Let $C$ denote the point $(0,1 / 2)$. Though the set $M_{2}$ is arc-wise connected and $M_{2}-C$ is an open connected subset of $M_{2}$, the points $O$ and $B$ cannot be joined by a simple arc which is a subset of $M_{2}-C$.

Sierpinski has shown $\S$ that a continuous curve may be characterized as a bounded continuum $M$ such that, for every positive number $\epsilon, M$ is the sum of a finite collection of closed and connected point sets each of which is of diameter less than $\epsilon$.

Thus we have five characterizations of the notion continuous curve, that of Jordan (or perhaps it should be called a modificontinuous curves, this Bulletin, vol. 23 (1917), pp. 233-236. This paper was presented to the American Mathematical Society, October 28, 1916.

* A point set $M$ is said to be arc-wise connected if every two distinct points of $M$ are the extremities of a simple continuous arc which lies wholly in $M$.

$\dagger$ R. L. Moore, Concerning continuous curves in the plane, MathemaTISCHE ZEITSCHRIFT, vol. 15 (1922), pp. 254-260. The point set $K$ is said to be an open subset of the set $M$ if $K$ is a subset of $M$ and $M-K$ is either vacuous or closed. A maximal connected subset of a point set $K$ is a connected subset of $K$ which is not a proper subset of any other subset of $K$.

$\ddagger$ This result forms a part of Mr. Wilder's dissertation for the degree of Ph.D. at the University of Texas.

$\S \mathrm{W}$. Sierpinski, Sur une condition pour qu'un continu soit une courbe jordanienne, Fundamenta Mathematice, vol. 1 (1920), pp. 44-60. 
cation of that of Jordan), that of Schoenflies, that of Hahn and Mazurkiewicz, that of Sierpinski, and lastly a characterization whose sufficiency and necessity have been established by Mr. Wilder and by the author, respectively. Of these characterizations, all but that of Schoenflies hold good in space of any number of dimensions.

2. The Relation of a Continuous Curve to its Complementary Domains, in Space of two Dimensions. Returning to Schoenflies' characterization, we may picture any continuous curve in the plane as the point set complementary to a finite or countably infinite set of simply connected domains such that (a) one of these domains is unbounded and contains, as a subset, the exterior of some circle which encloses the continuous curve, $(b)$ for every positive number $\epsilon$ there are not more than a finite number of these domains of diameter greater than $\epsilon$, (c) the boundary of each of these domains is, at each of its points, accessible from all sides with respect to that domain. Miss M. Torhorst has shown that the boundary of every complementary domain of a continuous curve is itself a continuous curve.* I have recently found that the outer boundary of every bounded complementary domain of a continuous curve is a simple closed curve and that if two points are separated from each other by a continuous curve $M$ then they are also separated by some simple closed curve which is a subset of $M . \dagger$ From this result, with the aid of the fact $\ddagger$ that every simple closed curve separates the plane, it follows that in order that a non-dense continuous curve

* Cf. Marie Torhorst, Ueber den Rand der einfach zusammenhängenden ebenen Gebiete, Mathematische Zeitschrift, vol. 9 (1921), pp. 44-65. In his thesis Mr. Wilder has shown that, indeed, every closed and connected subset of such a boundary is a continuous curve. This result extends, to the case of any such boundary, a proposition previously established by Mazurkiewicz for the case of a continuous curve which contains no simple closed curve. Cf. S. Mazurkiewicz, Un théorème sur les lignes de Jordan, Fundamenta Mathematice, vol. 2 (1921), pp. 119-130.

$\dagger$ Concerning continuous curves in the plane, loc. cit.

$\$$ Cf. O. Veblen, Theory of plane curves in non-metrical analysis situs, Transactions of this Society, vol. 6 (1905), pp. 107-112; and L. E. J. Brouwer, Beweis des Jordanschen Kurvensatzes, Mathematische Annalen, vol. 69 (1910), pp. 169-175. 
should separate the plane it is necessary and sufficient that it should contain a simple closed curve.

I will say that a point set $M$ has property $S$ if, for every positive number $\epsilon, M$ is the sum of a finite number of connected subsets all of diameter less than $\epsilon$. As applied to closed (and bounded) point sets this property is * equivalent to that of connectedness im kleinen. But, as applied to point sets which are not necessarily closed, it is $\dagger$ stronger than that of connectedness im kleinen and weaker than that of uniform $\ddagger$ connectedness im kleinen. Every domain is connected im kleinen. In order that a bounded and simply connected domain should have a continuous curve as its boundary it is $\S$ necessary and sufficient that it should have property $S$, while in order that it should have a simple closed curve as its boundary it is necessary and sufficient that it should have the stronger property of being uniformly connected im kleinen.\|

In his thesis Mr. Wilder makes a detailed study of the continuous curve which is the boundary of a domain. He has found that in order that the boundary $B$ of a simply connected domain should be a continuous curve it is necessary and sufficient that every connected subset of $B$ should be arc-wise connected.

3. A Characterization of Continua which are not Continuous Curves. I have found the following theorem very useful in the study of continuous curves.

Theorem. In order that a bounded continuum $M$ should fail to be a continuous curve, it is necessary and sufficient that there should exist two concentric circles $k_{1}$ and $k_{2}$ and a countable

* Cf. W. Sierpinski, loc. cit.

† Cf. my paper, Concerning continuous curves in the plane, loc. cit.

$\ddagger$ A point set $M$ is said to be uniformly connected im kleinen if, for every positive number $\epsilon$, there exists a positive number $\delta_{\epsilon}$ such that if $X$ and $Y$ are two points of $M$ at a distance apart less than $\delta_{\epsilon}$ then $X$ and $Y$ lie in some connected subset of $M$ of diameter less than $\epsilon$. Cf. Hans Hahn and S. Mazurkiewicz, loc. cit.

$\S$ R. L. Moore, loc. cit.

|| See my paper, A characterization of Jordan regions by properties having no reference to their boundaries, Proceedings of the National Academy, vol. 4 (1918), pp. 364-370. 
infinity of continua $\bar{M}, M_{1}, M_{2}, M_{3}, \cdots$, such that (1) each of these continua is a subset of $M$ and contains at least one point on $k_{1}$ and at least one point on $k_{2}$, and is a subset of the point set $H$ which is composed of the two circles $k_{1}$ and $k_{2}$ together with all those points of the plane which lie between these circles, (2) no two of these continua have a point in common, and, indeed, no one of them is a proper subset of any connected point set which is common to $M$ and $H$, (3) the set $\bar{M}$ is the sequential limiting set of the sequence of sets $M_{1}, M_{2}, M_{3}, \ldots,{ }^{*}$ (4) there exists a connected subset of $M$ which contains all the sets of the sequence $M_{1}, M_{2}, M_{3}, \cdots$, but which contains no point of the greatest connected point set which is common to $\bar{M}$ and $H$.

For an indication of a proof of all but part (4) of this theorem see my papers, Continuous sets that have no continuous sets of condensation, and $A$ characterization of Jordan regions by properties that have no reference to their boundaries. $\dagger$ The important part (4) has been supplied recently by Mr. Wilder. With its aid he established the above-mentioned theorem that in order that a bounded continuum $M$ should be a continuous curve it is sufficient that every maximal connected subset of an open subset of $M$ should be arc-wise connected. I have made use of parts (1), (2), and (3) on several occasions.

4. A Continuous Curve in the Rôle of a Space. It is of interest to note that if the set of points $S$ which constitute a continuous curve is viewed as a space and certain terms are properly defined, then many of the theorems which hold true in ordinary space continue to hold true in $S$. For each point

* The point set $M$ is said to be the limiting set of the sequence of point sets $M_{1}, M_{2}, M_{3}, \cdots$ provided that (a) each point of $M$ is the sequential limit point of an infinite subsequence of some sequence of points $P_{1}, P_{2}$, $P_{3}, \cdots$ such that, for every $n, P_{n}$ belongs to $M_{n}$, (b) if $P_{1}, P_{2}, P_{3}, \cdots$ is a sequence of points such that, for every $n, P_{n}$ belongs to $M_{n}$, then $M$ contains the sequential limit point of every subsequence of $P_{1}, P_{2}, P_{3}, \cdots$ that has a sequential limit point. If the further condition is satisfied that every infinite subsequence of the sequence $M_{1}, M_{2}, M_{3}, \cdots$ has the same limiting set $M$, then $M$ is said to be the sequential limiting set of the sequence $M_{1}, M_{2}, M_{3}, \cdots$.

$\dagger$ This Bulletin, vol. 25 (1919), pp. 174-176; and Proceedings of the National ACademy, loc. cit. 
$P$ in $S$ and each positive number $\epsilon$, let $R_{P \epsilon}$ denote the set of all those points of $S$ which lie, together with $P$, in a connected subset of $S$ which lies wholly within a circle with center at $P$ and radius equal to $\epsilon$. For every $P$ and $\epsilon$ the point set $R_{P \epsilon}$ will be called a region with respect to $S$. With the use of the fact that $S$ is connected im kleinen, it may be seen that a point $X$ in $S$ is a limit point of a point set $M$ which lies in $S$ if and only if every region (with respect to $S$ ) which contains $X$ contains also a point of $M$ distinct from $X$. It follows that if $S$ is identified with the $S$ of my set $\Sigma_{1}$ of axioms * for plane analysis situs then the point $X$ is a limit point of $M$ in the ordinary sense if and only if it is a limit point of $M$ in the sense defined in F. A. With the help of this fact it may be seen that Axioms 1,2, and 4, and a number of the theorems of that paper hold true in the space $S$, the terms (other than region) that are used in the statement of those axioms and theorems being given meanings which are ordinarily attached to them when $S$ is considered as a subset of ordinary space. Thus, corresponding to Theorem 15 of F. A., we have the previously mentioned theorem that every two points of a connected open subset $\dagger$ of a continuous curve can be joined by a simple continuous arc lying wholly in that subset.

In his thesis, in addition to other results in this connection, Mr. Wilder has established the following theorem.

Theorem. If $S$ is a continuous curve in space of two dimensions, $D$ is a domain with respect to $S, B$ is the boundary of $D$ with respect to $S$, and $P$ is a point of $D$, then in order that $P$ should be accessible, in $S$, from every point of $D$ it is sufficient either (a) that B should be connected im kleinen, or (b) that B should contain no continuum of condensation $\ddagger$ which contains $P$.

5. The Non-Cut Points of a Continuous Curve. A point $P$ of

* On the foundations of plane analysis situs, Transactions of THIS SocIETy, vol. 17 (1916), pp. 131-164. This paper will be referred to hereafter as F. A.

$\dagger$ A connected open subset of $S$ is called a domain in F. A., and such a subset of a continuous curve $S$ may be called a domain with respect to $S$.

$\ddagger$ A sub-continuum $K$ of a point set $M$ is said to be a continuum of condensation of $M$ if every point of $K$ is a limit point of $M-K$. 
a connected point set $M$ is said to be a non-cut point of $M$ or a cut point of $M$ according as $M-P$ is or is not connected. A cut point of $M$ is said to disconnect $M$ in the strong sense (or, merely, to disconnect $M$ ). A point set $M$ is said to be connected in the strong sense if every two points of $M$ lie together in some closed and connected subset of $M$. If $M-P$ is not connected in the strong sense then $P$ is said to disconnect $M$ in the weak sense. According to this definition, if a point $P$ disconnects a point set $M$ in the strong sense then it also disconnects it in the weak sense; but the converse is not always true. By Theorem A of $\S 1$, if $M$ is a continuous curve, then it is true, conversely, that if $P$ disconnects $M$ in the weak sense it also disconnects it in the strong sense.

In my paper Concerning simple continuous curves, ${ }^{*}$ I proved that if a bounded continuum $M$ does not contain more than two non-cut points then it is a simple continuous arc. But a simple continuous arc contains two non-cut points, namely its end-points. Thus we have the following theorem.

Theorem C. Every bounded continuum contains at least two non-cut points (i.e., two points neither of which disconnects it). $\dagger$

In his paper, Un théorème sur les lignes de Jordan, $\ddagger$ Mazurkiewicz has established the following theorems.

THeOREM $\overline{\mathrm{C}}$. Every continuous curve contains at least two points neither of which disconnects it in the weak sense.

Theorem D. If $M$ is a continuous curve which contains a simple closed curve $k$ then $k$ does not contain an uncountable set of points each of which disconnects $M$ in the weak sense.

Theorem $\mathrm{C}$ is a logical consequence of Theorems $\mathrm{A}$ and $\mathrm{C}$.

Let $\mathrm{D}^{\prime}$ denote the theorem obtained from Theorem $\mathrm{D}$ by the substitution of the word strong instead of the word weak. I have recently established the following theorems, $\S$ of which

* Loc. cit. Concerning the close relationship between this result and a result announced sometime before by Janiszewski see page 340 .

$\dagger J$. R. Kline has shown that if a continuum has no non-cut points it is connected im kleinen. Cf. this Bulletin, vol. 27 (1921), p. 399. That such a continuum is unbounded follows from Theorem C.

$\ddagger$ Loc. cit.

$\S$ For proofs of the first three of these theorems see my paper, On the 
the first includes Theorem $\mathrm{D}^{\prime}$ as a special case but is much more general than Theorem $\mathrm{D}^{\prime}$.

Theorem $\overline{\mathrm{D}}$. No closed and connected point set $M$ (whether it be a continuous curve or not) contains a closed and connected subset $K$ (whether $K$ be a simple closed curve or not) which contains an uncountable set of points each of which disconnects* $M$ but not $K$.

THEOREM E. In order that a bounded continuum $M$ should be a continuous curve which contains no simple closed curve, it is necessary and sufficient that every sub-continuum of $M$ should contain uncountably many points each of which disconnects $M$ in the strong sense.

THEOREM F. In order that the continuous curve $M$ should contain no simple closed curve it is necessary and sufficient that if $K$ denotes the set of all those points of $M$ that are not cut points of $M$, then no subset of $K$ disconnects $\dagger M$, even in the weak sense.

THEorem G. $\ddagger$ In order that a bounded continuum $M$ should be a simple closed curve, it is necessary and sufficient that it should be disconnected by the omission of any two of its points.

cut points of continuous curves and of other closed and connected point sets, Proceedings of the National Academy, vol. 9 (1923), pp. 101-106.

* That this theorem does not remain true if the word disconnects is replaced by the phrase disconnects in the weak sense may be seen if one considers the example of a point set $M$ which is composed of a circle $K$ together with a suitably selected simple spiral which has every point of $K$ as a limit point. In this example, no point of $K$ disconnects $K$ either in the strong or in the weak sense but every point of $K$ disconnects $M$ in the weak (but not in the strong) sense. This example also shows that Mazurkiewicz' Theorem D would not remain true if the phrase continuous curve were replaced by the phrase bounded, closed, and connected point set.

† If $N$ is a subset of $M$ and $M-N$ is not connected then $N$ is said to disconnect $M$. If $M-N$ is not strongly connected then $N$ is said to disconneot $M$ in the weak sense.

$\ddagger$ Cf. my paper Concerning simple continuous curves, loc. cit. In a paper presented to the Society but, as far as I know, not as yet published, J. R. Kline has shown that in this theorem the stipulation that the set $M$ is bounded may be replaced by the stipulation that $M$ has no cut point. He also characterizes a simple closed curve as a continuum which is not disconnected by the omission of any proper connected subset. Cf. this Bulletin, vol. 27 (1921), p. 399, and vol. 28 (1922), p. 8. 
6. Concluding Remarks. Beyond certain propositions which hold true for both two and three dimensions, comparatively little is known concerning continuous curves in three-dimensional space. Various results obtained by Schoenflies, Miss Torhorst and others concerning the relation of a continuous curve to its complementary domains in the plane do not hold true in three-space.* For instance Schoenflies has shown that if a bounded plane continuum divides its plane into just two domains $S_{1}$ and $S_{2}$ such that every point of $M$ is accessible from both $S_{1}$ and $S_{2}$ then $M$ is a simple closed curve. In threedimensional space however there exists a bounded continuum $M$ which divides space into just two domains $S_{1}$ and $S_{2}$ such that (a) every point of $M$ is accessible from both $S_{1}$ and $S_{2}$ and $(b)$ the domain $S_{1}$ is uniformly connected im kleinen, but $M$ is neither a simple closed surface nor any sort of continuous curve. I have shown, $\dagger$ that such a point set $M$ would be a continuous curve if both $S_{1}$ and $S_{2}$ were uniformly connected im kleinen. It seems to me conceivable that some results concerning continuous curves and their complementary domains may be modified that they will hold true for threespace if, in addition to other changes, one replaces in certain connections the diameter of a point set by what I have called the two-dimensional extent of a point set.

Definition. A point set $M$ is said to have a two-dimensional extent greater than $e$ if there exists a right circular cylinder $\alpha$ with bases of diameter greater than $e$ such that (a) neither base of $\alpha$ contains a point of $M$, (b) $M$ contains at least one point in common with every simple continuous arc which has one end-point on one base of $\alpha$, and its other endpoint on the other base of $\alpha$ and lies, except for its end-points, wholly within $\alpha$.

Every simple closed curve in a space $S_{2}$ of two dimensions can be thrown into any other simple closed curve in $S_{2}$ by a continuous one to one reciprocal transformation of $S_{2}$ into

* See my paper, On the relation of a continuous curve to its complementary domains in space of three dimensions, loc. cit.

$\dagger$ Loc. cit. 
itself.* In other words in $S_{2}$ every two simple closed curves are equivalent to each other, in the strong sense, from the viewpoint of analysis situs. This proposition does not hold true for a space $S_{3}$ of three dimensions, as may be easily seen if one considers two simple closed curves in $S_{3}$ of which one is knotted and the other one is not. A knotted simple closed curve may be defined, from the viewpoint of analysis situs, as a simple closed curve (see definitions above) which is not a part of any simple closed $\dagger$ surface. It seems likely $(a)$ that there exists no knotted simple continuous arc, that is to say, no simple continuous arc which does not lie on some simple closed surface, and $(b)$ that any simple continuous arc in $S_{3}$ can be transformed into any other simple continuous arc in $S_{3}$ by a continuous one to one reciprocal transformation of $S_{3}$ into itself. But, as far as I know, no one has ever proved this. Indeed, as far as I know, there is nowhere in the literature any indication that anyone has even considered the question whether, if $A B$ is an arc in a three-space $S$, and $X$ is a point of $S$ which does not belong to $A B$, then there exists an arc $X B$ which has in common with $A B$ only the point $B$. In other words, is an arc accessible at each of its ends from every point which does not lie on it?

A fruitful field for future investigation is afforded in that branch of analysis situs which has to do with various special types of continuous curves such as simple continuous arcs, simple closed curves and simple closed surfaces, in space of three dimensions. Little is known concerning the relation of such point sets to their complementary domains.

The University of Texas

* Cf. A. Schoenflies, Mathematische Annalen, vol. 62 (1906), p. 324. Also J. R. Kline, A new proof of a theorem due to Schoenflies, Procendings of the National ACADEMY, vol. 6 (1920), pp. 529-531.

$\dagger$ By a simple closed surface is meant a point set which is in one to one continuous correspondence with the surface of a sphere. For a topological characterization of such a point set, see the abstract of an unpublished paper by J. R. Kline and the author, this Bulletin, vol. 28 (1922), p. 380. As far as I know, there does not exist in the literature a proof that a simple closed surface is the image of a sphere under a one to one reciprocal continuous transformation of $S$ into itself. See, however, an abstract by J. W. Alexander, this Bulletin, vol. 28 (1922), p. 10. 